\title{
Patrones de sensibilidad de Staphylococcus aureus de la comunidad aislados de niños con infecciones de piel y partes blandas
}

\section{Sensitivity Patterns of Community-acquired Staphylococcus aureus Isolated from Skin and Soft Tissue in Children}

\author{
Gloria Celeste Samudio-Domínguez ${ }^{(1)}$, Lorena Bordón ${ }^{(1)}$, Natalia D`apollo ${ }^{(1)}$, Mercedes Martínez \\ Bareiro $^{(1)}$, Diego Benítez ${ }^{(1)}$
}

\section{RESUMEN}

Introducción: El Staphylococcus aureus ha experimentado durante las últimas décadas un cambio importante en la sensibilidad a la oxacilina en diferentes países. En Paraguay existen reportes de resistencia crecientes, por lo cual se hace imprescindibles conocer adecuadamente la tendencia en los patrones de resistencia a los antimicrobianos, pues el retraso en el inicio de una terapia adecuada y correcta, se asocia a una mayor morbilidad y mortalidad. Objetivo: Reportar el perfil de resistencia del S. aureus de población pediátrica del Hospital General de Barrio Obrero. Materiales y Métodos: Estudio, observacional, descriptivo, de corte transverso, realizado de enero 2012 a Julio de 2013 de muestras pediátricas no invasivas. Se utilizó equipo automatizado Vitek2 Compact (Bio merieux) para la identificación y el antibiograma. Resultados: Se aislaron 79 cepas de niños de 0 a 16 años, el sitio de aislamiento fue secreción purulenta. Resistencia a Oxacilina $70 \%$, Resistencia a Clindamicina 21\%, Resistencia inducible a Clindamicina 14\%. Sensibilidad $100 \%$ o cercana al 100\% para Ciprofloxacina, Rifampicina, Trimetropim, Levofloxacina, Tetraciclina, el MIC mas alto para vancomicina fue menor de 1. Conclusión: La elevada resistencia del $S$. aureus a la oxacilina en este hospital hace obligatorio el uso de drogas alternativas como terapia empírica, tales como la clindamicina y otros.

Palabras clave: Staphylococcus aureus, muestras no invasivas, oxacilina, resistencia.

\section{ABSTRACT}

Introduction: In recent decades Staphylococcus aureus has significantly changed its sensitivity to oxacillin in a number of countries. Reports of resistance have increased in Paraguay, making adequate knowledge of trends in antimicrobial resistance patterns vital, as delayed initiation of appropriate and adequate therapy is associated with greater morbidity and mortality. Objective: To report the resistance profile of $S$. aureus in the population of children seen at the Hospital General de Barrio Obrero, Paraguay. Materials and Methods: We conducted a cross-sectional observational and descriptive study of non-invasive samples taken from children between January 2012 and July 2013. The automated Vitek2 Compact (bioMerieux) was used for identification and the antibiogram. Results: We isolated 79 strains in samples taken from purulent discharge from children aged 0 to 16 . Resistance was found to oxacillin in $70 \%$, to clindamycin in $21 \%$, and inducible resistance to clindamycin in $14 \%$. Sensitivity was $100 \%$ or near $100 \%$ for ciprofloxacin, rifampin, trimethoprim, levofloxacin, and tetracycline, the highest minimum inhibitory concentration for vancomycin was less than 1 . Conclusion: The high resistance of $S$. aureus to oxacillin in our hospital makes the use of alternative drugs such as clindamycin or others obligatory in empirical therapy.

Keywords: Staphylococcus aureus, non-invasive samples, oxacillin, resistance.

\footnotetext{
1. Hospital General Barrio Obrero, Ministerio de Salud Pública y Bienestar Social. Asunción, Paraguay. Correspondencia: Dra. Gloria CelesteSamudio-Domínguez.E-mail: gsamudio.samudio@gmail.com Recibido: 12/05/2014; Aceptado: 07/09/2014. http://dx.doi.org/10.18004/ped.2015.abril.31-36 Los autores declaran que no existen conflictos de interés en el presente estudio.
} 


\section{INTRODUCCIÓN}

El Staphylococcus aureus es un microrganismo que comúnmente causa infecciones de piel y partes blandas. En la última década se ha observado un aumento global de las tasas de resistencia del mismo a la meticilina, hecho que ha ocurrido en muy corto periodo de tiempo ${ }^{(1)}$.

Los Staphylococcus aureus meticilino resistentes adquiridos en la comunidad (SAMR-CA) poseen en común con aquellos adquiridos en el hospital (SAMR-HA) su resistencia a la meticilina, sin embargo la patogénesis, epidemiología y manifestaciones clínicas pueden diferir. Los SAMRCA causan infecciones severas, incluyendo neumonía necrotizante, fascitis, púrpura fulminas y sepsis severa. Tiene una virulencia mayor que las cepas de SAMR-HA y que los Staphylococcus aureus meticilino sensibles, hecho aún no bien comprendido ${ }^{(2)}$.

Al parecer, la presencia de leucocidina de Panton Valentine (PVL) podría ser uno de los motivos de la expresión más severa de la enfermedad. El abordaje terapéutico dependerá de la extensión de la lesión, la afectación clínica del paciente y las alteraciones laboratoriales ${ }^{(3)}$.

En Paraguay existen diversos estudios de resistencia, la cual se ha ido incrementando a través de los años, como el trabajo de Román y col. que no reportaron SAMR en $2009^{(4)}$ y Sanabria y col. con reportes del $23 \%$ de resistencia a meticilina en un hospital de referencia de pacientes infectados en el mismo año ${ }^{(5)}$.

En el año 2012, el Dr. Rala reporta una resistencia mayor del 50\% en SAMR, aunque el trabajo no discrimina si los pacientes internados tienen enfermedad causada por SAMR-CAo-SAMR-HA ${ }^{(6)}$.

El objetivo de este trabajo es conocer el perfil de resistencias de los Staphylococcus aureus adquiridos en la comunidad, obtenidos de los cultivos de secreción purulenta de infección de piel y partes blandas de pacientes pediátricos internados en un hospital de segundo nivel.

\section{MATERIALES Y MÉTODOS}

Estudio observacional, descriptivo de frecuencias, realizado de enero de 2012 a julio de 2013. Se incluyeron al estudio todos los cultivos positivos Staphylococcus aureus aislados a partir de secreción purulenta tomada de piel y partes blandas de pacientes pediátricos a su ingreso al hospital. Para el cultivo y antibiograma se utilizó equipo automatizado Vitek2 Compact (Bio merieux). El trabajo de microbiología fue realizado por microbiólogos experimentados. En caso de duda de la sensibilidad las cepas fueron remitidas a un laboratorio de referencia para control externo. Para el análisis de datos se utilizó planilla Excel con aplicación bioestadística.

\section{RESULTADOS}

Se aislaron 79 cepas de Staphylococcus aureus de niños de 0 a 16 años, el promedio de edad fue de 5 años, con extremos de 1 mes a 15 años. La distribución de los pacientes, según el sexo fue de $43(54 \%)$ de sexo masculino y $36(46 \%)$ femenino.

De las 79 cepas incluidas al estudio, 55 (70\%) fueron resistentes a la oxacilina (Figura 1).

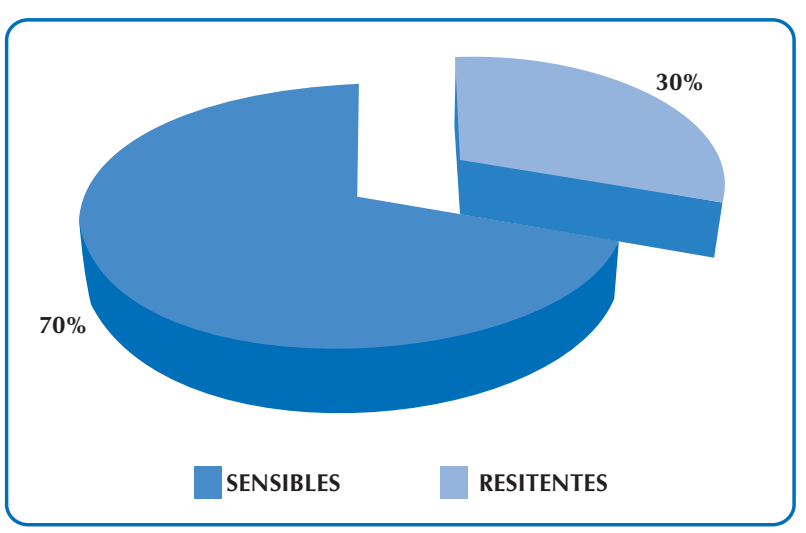

Figura 1. Resistencia a la oxacilina de los Staphylococcus aureus adquiridos en la comunidad, enero 2012 a julio 2013 (N=79).

También se midió resistencia a otros fármacos y se encontró las siguientes resistencias: $16(20 \%)$ a Clindamicina, $11(14 \%)$ con resistencia inducible a Clindamicina. Ninguna de las cepas sensibles a eritromicina presentó resistencia inducible a clindamicina.

La resistencia a gentamicina se presentó en 3 (4\%) de los casos. Quince $(19 \%)$ de las cepas fueron resistentes a la eritromicina (Figura 2). 


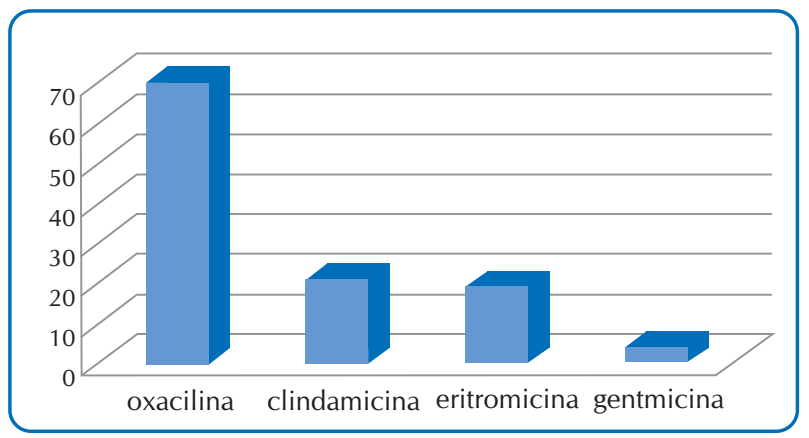

Figura 2. Resistencia del Staphylococcus aureus de la comunidad a diversos antimicrobianos, enero de 2012 a julio de 2013 ( $N=79$ ).

La resistencia de los Staphylococcus aureus fue de 1 $(1,2 \%)$ para ciprofloxacina, rifampicina, Trimetroprim-sulfametoxazol, Levofloxacina y Tetraciclina respectivamente.

No se encontró resistencia para vancomicina, tigeciclina, quinupristin-dalfopristin o linezolid.

Los niveles de la Concentración Inhibitoria Mínima (MIC) para la vancomicina, de importancia para la evolución clínica de la infección ${ }^{(7-11)}$, fueron medidos para todos los gérmenes aislados y se encontró que de las 55 cepas resistentes a la oxacilina, 9 (16\%) tuvieron MIC de 1 y en los restantes 46 (84\%) el MIC fue de 0,5 (Figura 3).

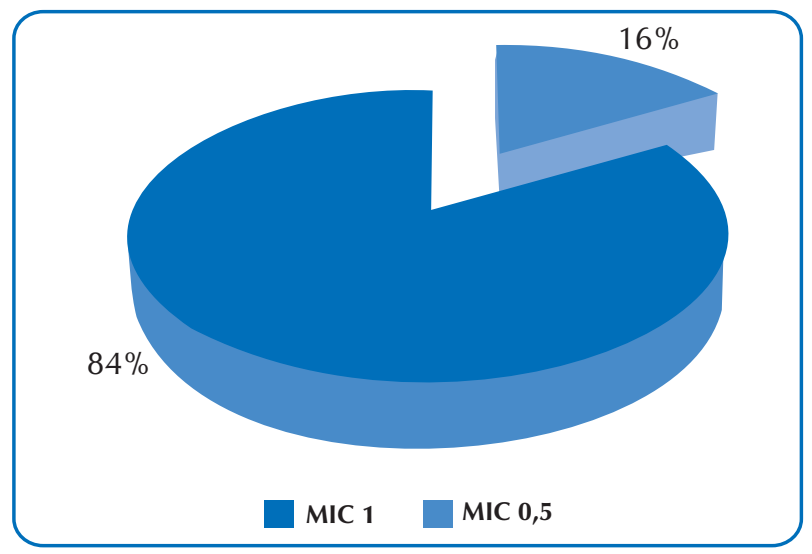

Figura 3. Niveles de MIC para vancomicina en los Staphylococcus aureus resistentes a meticilina adquiridos en la comunidad (SAMR-CA), enero 2012 a julio 2013 ( $\mathrm{N=55).}$

\section{DISCUSIÓN}

El Staphylococcus aureus (SA) es un patógeno aislado frecuentemente, sobre todo en infecciones de piel y partes blandas. Tiene además la patogenicidad suficiente para causar invasiones potencialmente fatales. La resistencia de este germen a las oxacilina ha ido variando a través de los años, demostrando una tendencia ascendente, estableciendo nuevas reglas a la hora de elegir la mejor terapia empírica inicial para tratar la afección causada por el mismo ${ }^{(2-}$ 6).

Diversos estudios a lo largo del mundo reportan resistencias crecientes ${ }^{(12-15)}$. En nuestro país, varios investigadores han reportado datos de sensibilidad y resistencia de este germen de muestras invasivas con niveles de resistencias que van hasta cercanas al $30 \%$ o $50 \%^{(4-6)}$ aunque la mayor parte de los aislamientos se refieren a los Staphylococcus aureus sin una diferencia clara de cepas adquiridas en la comunidad o en el ámbito hospitalario ${ }^{(16-18)}$.

En casos de resistencia a la oxacilina, el uso de vancomicina debe ser planteado. Sin embargo, existen peligros inherentes a su uso, desde los efectos adversos hasta la modificación de la flora e inducción de resistencias en gérmenes tales como los enterococos vancomicino resistentes ${ }^{(19,20)}$.

Por este motivo, probablemente sea más prudente plantear como terapia empírica inicial la clindamicina, siempre que los niveles de resistencia y estabilidad del paciente lo permitan ${ }^{(21)}$.

Este último, si bien es un fármaco bacteriostático tiene la ventaja de poseer efecto inmunomodulador, sin embargo ha sido relacionado en el pasado con la aparición de casos de colitis pseudomembranosa, por lo que el uso debe ser vigilado a fin de manejar los efectos adversos en forma pronta y adecuada ${ }^{(22-24)}$.

La resistencia inducible a la clindamicina en las cepas de SAMR-CA debe tenerse en cuenta, para evitar posibles fallos terapéuticos. Se debería tamizar de manera obligatoria esta resistencia a todos los estafilococos que presenten el fenotipo de resistencia a eritromicina y sensibilidad a clindamicina in vitro para evitar fracasos terapéuticos, teniendo en cuenta que la clindamicina es un antibiótico de bajo costo y una excelente alternativa de tratamiento en infecciones cutáneas y osteoarticulares ${ }^{(25-29)}$.

En nuestras cepas se encontró $14 \%$ de resistencia inducible; sin embargo ninguna cepa resistente a la eritromicina fue sensible a clindamicina. Estos porcentajes se encuentran dentro de lo reportado 
por diversos autores $^{(30-31)}$.

Los casos de SAMR con sensibilidad intermedia a la vancomicina (VISA) han sido reportado en diversos $\operatorname{artículos}^{(32-37)}$.

Nosotros no hemos tenido ningún caso. Los niveles de MIC para la vancomicina en los SAMR son importantes para la evolución del paciente, ya que existen reportes que demuestran que $\mathrm{MIC} \geq 1,5$ se asocian con mala evolución del paciente, cuando se trata de infecciones invasivas ${ }^{(38-40)}$.

En nuestra serie, la mayoría de las cepas tienen MIC de 0,5 , esto lleva a dos vertientes de pensamiento: tienen este MIC por ser cepas no invasivas o porque aún están en los inicios del proceso evolutivo de resistencia? Esta pregunta no podrá ser contestada aún por los autores de este reporte.

El abordaje actual del tratamiento empírico inicial de las infecciones de piel y partes blandas establece que si las lesiones son mayores de 5 centímetros y/o el paciente tiene datos de respuesta inflamatoria

\section{REFERENCIAS}

1. Tarai B, Das P, Kumar D. Recurrent challenges for clinicians: emergence of Methicillin-Resistant Staphylococcus aureus, vancomycin resistance, and current treatment pptions. J Lab Physicians. 2013;5(2):71-78.

2. Singer AJ, Talan DA. Management of skin abscesses in the era of Methicillin-Resistant Staphylococcus aureus. N Engl J Med. 2014;370:1039-47. DOI:10.1056/ NEJMra1212788

3. Kowalsky TJ, Berbari EF, Osmon DR. Epidemiology, treatment, and prevention of community-acquired methicillin-resistant Staphylococcus aureus infections. Mayo Clin Proc. 2005;80(9):1201-08.

4. Roman RO, SamudioD GC, Carnibella M. Infecciones por Staphylococcus aureus en pacientes en edad pediátrica internada en el Hospital Nacional de Itaugua [tesis]. Itauguá: Hospital Nacional de Itauguá; 2009.

5. Sanabria G, Araya S, Apodaca S, Ayala de PE, Lovera D, Arbo A. Infección de piel y partes blandas causadas por Staphylococcus aureus adquirido en la comunidad en niños hospitalizados en un centro de referencia en Paraguay: 8 años de vigilancia. Asunción: Instituto de Medicina Tropical; 2009. sistémica y/o alteración de laboratorio con datos de infección, el paciente debe ser internado y tratado por vía endovenosa. Los que no cumplan esos criterios pueden ser enviados a domicilio, luego de drenada la colección, con antibiótico vía oral, o aún sin antibióticos ${ }^{(41-43)}$.

Una de las debilidades de este trabajo es que se midió solo las sensibilidades de los gérmenes, sin correlacionar con los datos clínicos, demográficos o de evolución de los pacientes. Esto, sin embargo, puede servir para, a partir de estos datos, realizar una investigación que abarque tanto la microbiología como la clínica.

\section{CONCLUSIÓN}

La elevada resistencia del Staphylococcus Aureus a la oxacilina en nuestro hospital hace obligatorio el uso de drogas alternativas como terapia empírica, tales como la clindamicina y vancomicina.

6. Irala JD. Progresión de la resistencia de Staphylococcus aureus a Oxacilina en el Instituto de Medicina Tropical de Asunción, Paraguay. Rev Inst Med Trop. 2013;8(1):18-25.

7. Nakaminami $\mathrm{H}$, Noguchi N, Ito A, Ikeda M, Utsumi K, Maruyama H, Sakamoto $H$, Senoo M, Takasato $Y$, Nishinarita S. Characterization of methicillin-resistant Staphylococcus aureus isolated from tertiary care hospitals in Tokyo, Japan. J Infect Chemother. 2014;20(8):512-15. DOI: 10.1016/j.jiac.2014.03.006. Epub 2014 Apr 21.

8. Lee JY, Chong YP, Kim T, Hong HL, Park SJ, Lee ES, Kim $\mathrm{MN}$, Kim SH, Lee SO, Choi SH, Woo JH, Kim YS. Bone and joint infection as a predictor of community-acquired methicillin-resistant Staphylococcus aureus bacteraemia: a comparative cohort study. J Antimicrob Chemother. 2014;69(7):1966-71. DOI: 10.1093/jac/dku076. Epub 2014 Apr 2.

9. Larru B, Gerber JS. Cutaneous bacterial infections caused by Staphylococcus aureus and Streptococcus pyogenes in infants and children. Pediatr Clin North Am. 2014;61(2):457-78. DOI: 10.1016/j.pcl.2013.12.004. Epub 2014 Jan 23. 
10. Guss J, Kazahaya K. Antibiotic-resistant Staphylococcus aureus in community-acquired pediatric neck abscesses. Int J Pediatr Otorhinolaryngol. 2007;71(6):943-8.

11. Le J, Lieberman JM. Management of communityassociated methicillin-resistant Staphylococcus aureus infections in children. Pharmacotherapy. 2006;26(12):1758-70.

12. Morales GI, Yaneth MC, Chávez KM. Caracterización de la resistencia in vitro a diferentes antimicrobianos en cepas de Staphylococcus spp. en una institución hospitalaria de la ciudad de Valledupar entre enero y julio de 2009. Rev Cienc Salud. 2012;10(2):169-77.

13. Deurenberg RH, Stobberingh EE. The evolution of Staphylococcus aureus. Infect Genet Evol. 2008;8(6):747-63. DOI: 10.1016/j.meegid.2008.07.007

14. Buescher ES. Community-acquired methicillinresistant Staphylococcus aureus in pediatrics. Curr Opin Pediatr. 2005;17(1):67-70.

15. Miranda Novales MG. Resistencia antimicrobiana del Staphylococcus aureus en México. Bol Med Hosp Infant Mex [Internet]. 2011 [citado 2014 May 11]; 68(4):262-70. Disponible en: http://www.scielo.org.mx/scielo.php?script $=$ sci_arttext\&pid=S1665-11462011000400003\&lng=es

16. Mayor L, Ortellado J, Menacho C, Lird G, Courtier C, Gardon C, Meugnier H, Bes M, Vandenesch F, Etienne J. Molecular characterization of methicillin-resistant Staphylococcus aureus isolates collected in Asunción, Paraguay. J Clin Microbiol. 2007;45(7):2298-300.

17. Soilan B, Gómez G, Ortellado J, Lird G, Marín M, Heinichen O, Almada P, Galeano A, Vera R, Ramos C, Mayor M, Ayala R. Portación de Staphylococcus aureus en pacientes dializados del servicio de Nefrología HC-IPS. Rev Inst Med Trop. 2011;6(Supl.):44.

18. Guillén R, Basulado W, Castro H, Campuzano de Rolón A, Macchi M, Ortellado J, Almada P, Quiñonez B, Arguello R, Rodríguez M, Palacios M, Grau L, Velázquez G, Espínola C, Samudio G, Gómez G, Carpinelli L, Russomando G. Staphylococcus aureus adquiridos en la comunidad: caracterización clínica, fenotípica y genotípica de aislados en niños que concurren a hospitales de referencia de Asunción y Dpto. Central. Rev Inst Med Trop. 2011; 6(Supl.):45.

19. Fica A, Jemenao MI, Bilbao P, Ruiz G, Sakurada A, Pérez de Arce E, Zuñiga I, Gompertz M. Emergencia de infecciones por enterococcus sp resistente a vancomicina en un hospital universitario en Chile. Rev Chil Infectol [Internet]. 2007 Dic [citado 2014 Mayo 11]; 24(6):462-71. Disponible en: http://www.scielo.cl/scielo.php?script=sci_ arttext\&pid=S0716-10182007000600006\&lng=es. http://dx.doi.org/10.4067/S0716-10182007000600006.
20. Braun S, Fica A, Andrighetti D, Porte L, Triantafilo V. Estudio de factores de riesgo para colonización por enterococo resistente a vancomicina en el Hospital Militar de Santiago. Rev Chil Infectol [Internet]. 2009 Ago [citado 2014 May 11];26(4):374-75. Disponible en: http://www.scielo.cl/scielo.php?script=sci_arttext\&pid=S $0716-10182009000500014$ \& $1 \mathrm{ng}=\mathrm{e} \mathrm{s}$ http://dx.doi.org/10.4067/S0716-10182009000500014

21. Forcade NA, Parchman ML, Jorgensen JH, Du LC, Nyren NR, Treviño LB, Peña J, Mann MW, Muñoz A, Treviño SB, Mortensen EM, Wickes BL, Pollock BH, Frei CR. Prevalence, severity, and treatment of communityacquired methicillin-resistant Staphylococcus aureus (CAMRSA) skin and soft tissue infections in 10 medical clinics in Texas: a South Texas Ambulatory Research Network (STARNet) study. J Am Board Fam Med. 2011;24(5):543-50. DOI: $10.3122 / j a b f m .2011 .05 .110073$

22. Ogami $\mathrm{N}$, , Ishimaru $\mathrm{T}$, , Matsubara N, Ueno T, Asano I. Is Clostridium difficile infection influenced by antimicrobial use density in wards? Jpn J Antibiot. 2013;66(2):87-95.

23. Hugo H, Dornbusch K, Sterner G. Studies on the clinical efficacy, serum levels and side effects of clindamycin phosphate administered intravenously. Scand J Infect Dis. 1977;9(3):221-26.

24. Dial S, Alrasadi K, Manoukian C, Huang A, Menzies D. Risk of Clostridium difficile diarrhea among hospital inpatients prescribed proton pump inhibitors: cohort and case-control studies. CMAJ. 2004;171(1):33-38.

25. Lall M, Sahni AK. Prevalence of inducible clindamycin resistance in Staphylococcus aureus isolated from clinical samples. Med J Armed Forces India. 2014;70(1):43-47. DOI: 10.1016/j.mjafi.2013.01.004. Epub 2013 May 8.

26. Hersh AL, Weintrub PS, Cabana MD. Antibiotic selection for purulent skin and soft-tissue infections in ambulatory care: a decision-analytic approach. Acad Pediatr. 2009;9(3):179-84. DOI: 10.1016/j.acap.2009.02.002.

27. Napolitano LM. Early appropriate parenteral antimicrobial treatment of complicated skin and soft tissue infections caused by methicillin-resistant Staphylococcus aureus. Surg Infect (Larchmt). 2008;9(Suppl 1):s17-27. DOI: 10.1089/sur.2008.063.supp.

28. Teng CS, Lo WT, Wang SR, Tseng MH, Chu ML, Wang CC. The role of antimicrobial therapy for treatment of uncomplicated skin and soft tissue infections from community-acquired methicillin-resistant Staphylococcus aureus in children. J Microbiol Immunol Infect. 2009;42(4):324-28.

29. Geng W, Yang Y, Wang C, Deng L, Zheng Y, Shen X. Skin and soft tissue infections caused by communityassociated methicillin-resistant Staphylococcus aureus 
among children in China. Acta Paediatr. 2010;99(4):575-80. DOI: 10.1111/j.1651-2227.2009.01645.x.

30. Montoya I, Mira M, Álvarez I, Cofré J, Cohen J, Donoso G, Torres JP. Resistencia inducible a clindamicina en Staphylococcus aureus meticilino resistente. Rev Chil Pediatr. 2009;80(1):48-53.

31. Merino-Díaz L, Cantos de la Casa A, Torres-Sánchez MJ, Aznar-Martín J. Detección de resistencia inducible a clindamicina en aislados cutáneos de Staphylococcus spp. por métodos fenotípicos y genotípicos. Enfermedades Infecciosas y Microbiología Clínica. 2007;25(2):77-81.

32. Appelbaum PC. Reduced glycopeptide susceptibility in methicillin-resistant Staphylococcus aureus (MRSA). Int J Antimicrob Agents. 2007;30(5):398-408.

33. Khatib R, Jose J, Musta A, Sharma M, Fakih MG, Johnson LB, Riederer $K$, Shemes S. Relevance of vancomycin-intermediate susceptibility and heteroresistance in methicillin-resistant Staphylococcus aureus bacteraemia. J Antimicrob Chemother. 2011;66(7):1594-99. DOI: 10.1093/jac/dkr169.

34. Martínez-Aguilar G, Hammerman WA, Mason EO Jr, Kaplan SL. Clindamycin treatment of invasive infections caused by community-acquired, methicillin-resistant and methicillin-susceptible Staphylococcus aureus in children. Pediatr Infect Dis J. 2003;22(7):593-98.

35. Khatib R, Jose J, Musta A, Sharma M, Fakih MG, Johnson LB, Riederer K, Shemes S. Relevance of vancomycin-intermediate susceptibility and heteroresistance in methicillin-resistant Staphylococcus aureus bacteraemia. J Antimicrob Chemother. 2011 Jul;66(7):1594-9.

36. Musta AC, Riederer K, Shemes S, Chase P, Jose J, Johnson LB, Khatib R. Vancomycin MIC plus heteroresistance and outcome of methicillin-resistant Staphylococcus aureus bacteremia: trends over 11 years. J Clin Microbiol. 2009;47(6):1640-44.
37. van Hal SJ, Lodise TP, Paterson DL. The clinical significance of vancomycin minimum inhibitory concentration in Staphylococcus aureus infections: a systematic review and meta-analysis. Clin Infect Dis. 2012;54(6):755-71. DOI: 10.1093/cid/cir935.

38. Chen SY, Hsueh PR, Chiang WC, Huang EP, Lin CF, Chang CH, Chen SC, Chen WJ, Chang SC, Lai MS, Chie WC. Predicting high vancomycin minimum inhibitory concentration isolate infection among patients with community-onset methicillin-resistant Staphylococcus aureus bacteremia. J Infect. 2014;69(3):259-65. DOI: 10.1016/j.jinf.2014.04.007. Epub 2014 May 4.

39. Lubin AS, Snydman DR, Ruthazer R, Bide P, Golan Y. Predicting high vancomycin minimum inhibitory concentration in methicillin-resistant Staphylococcus aureus bloodstream infections. Clin Infect Dis. 2011;52(8):997-1002. DOI: 10.1093/cid/cir118.

40. Honda H, Doern CD, Michael-Dunne W Jr, Warren DK. The impact of vancomycin susceptibility on treatment outcomes among patients with methicillin resistant Staphylococcus aureus bacteremia. BMC Infect Dis. 2011;11:335. DOI: 10.1186/1471-2334-11-335.

41. Walraven CJ, North MS, Marr-Lyon L, Deming P, Sakoulas G, Mercier RC. Site of infection rather than vancomycin MIC predicts vancomycin treatment failure in methicillin-resistant Staphylococcus aureus bacteraemia. J Antimicrob Chemother. 2011;66(10):2386-92. DOI: 10.1093/jac/dkr301.

42. Meddles-Torres C, Hu S, Jurgens C. Changes in prescriptive practices in skin and soft tissue infections associated with the increased occurrence of community acquired methicillin resistant Staphylococcus aureus. J Infect Public Health. 2013;6(6):423-30. DOI: 10.1016/j.jiph.2013.04.010.

43. Zervos M. Treatment options for uncomplicated community-acquired skin and soft tissue infections caused by methicillin-resistant Staphylococcus aureus: oral antimicrobial agents. Surg Infect (Larchmt). 2008;9(Suppl 1):s29-34. DOI: 10.1089/sur.2008.065.supp. 